\title{
A comparison of Newtonian and non-Newtonian models for pulsatile blood flow simulations
}

\begin{abstract}
Mathematical modeling of blood flows in the arteries is an important and challenging problem. This study compares several non-Newtonian blood models with the Newtonian model in simulating pulsatile blood flow through two three-dimensional models of an arterial stenosis and an aneurysm. Four non-Newtonian blood models, namely the Power Law, the Casson, the Carreau, and the Generalized Power Law, as well as the Newtonian model of blood viscosity, are used to investigate the flow effects induced by these different blood constitutive equations. The aim of this study is three-fold: firstly, to investigate the variation in wall shear stress in an artery with a stenosis or aneurysm at different flow rates and degrees of severity; secondly, to compare the various blood models and hence quantify the differences between the models and judge their significance; and lastly, to determine whether the use of the Newtonian blood model is appropriate over a wide range of shear rates.
\end{abstract}

Keywords: aneurysm; blood; fluid flows; non-newtonian; pulsatile; simulations; stenosis.

*Corresponding author: Iqbal Husain, Department of Mathematics, Luther College, University of Regina, Regina, Saskatchewan, Canada S4S 0A2, e-mail: Iqbal.Husain@uregina.ca Fotini Labropulu, Chris Langdon and Justin Schwark: Department of Mathematics, Luther College, University of Regina, Regina, Saskatchewan, Canada S4S 0A2

\section{Introduction}

The mathematical modeling and numerical simulations of the human cardiovascular system is playing an increasingly important role in our understanding of the cause and development of cardiovascular diseases. These diseases are a major cause of mortality and have a substantial impact on the cost of healthcare in many developed and developing countries. As a result, there is a growing demand from the medical community for scientifically rigorous quantitative investigations of these diseases which has provided a major impetus to the development of mathematical models and numerical methods for the computer simulations of various anomalies in the human cardiovascular system. However, the circulatory system is highly integrated and modeling its various functions is a very challenging problem, physically, mathematically and computationally, requiring the consideration of many fundamental issues in all three areas.

Since the hemodynamic hypotheses of atherosclerosis were first formulated several decades ago, flow imaging and computing have contributed significantly to our knowledge of how blood really flows in large arteries prone to atherosclerosis [1, 2]. By the mid-1980s, computational fluid dynamics (CFD) emerged as a practical tool for examining local hemodynamics at a level of detail thus far impossible with experimental techniques, which made it possible to consider increasingly sophisticated blood flow indicators [3]. By the mid-1990s, it became possible to consider the integration of medical imaging and CFD as a means of investigating and exploiting the relationship between local hemodynamics and vascular dysfunctions in a subject-specific manner [4]. At present, for "routine" investigations of large arteries, and in particular parametric studies of geometric or flow related factors, CFD has almost completely replaced traditional engineering flow measurement techniques such as laser Doppler velocimetry and particle imaging velocimetry. This may be due to the relative ease with which input parameters can be varied in CFD, particularly in the case of pulsatile flows, and the desire to quantify flow parameters such as shear stress gradients, which can tax the resolution limits of experimental flow measurements. However, CFD remains relatively immature in analyzing models with moving boundaries, fine embedded devices, and especially where flow instabilities or turbulence may occur [5].

While lumped parameter and linear and nonlinear one-dimensional wave propagation methods received much attention from the 1950s through to the 1980s, CFD methods based on solving the three-dimensional equations of blood flow have had a dominant influence on current research efforts to quantify hemodynamic conditions in arteries. CFD techniques can be used to provide detailed data on flow conditions unattainable with simpler, onedimensional and lumped parameter methods, and difficult to obtain from experimental measurements alone. Briefly, CFD solves the equations governing fluid flow by 
breaking up a complex geometry into many smaller but simpler shapes. All that is required is a description of the geometry and information about the flow entering and leaving the model boundaries. The result is a detailed representation of the velocity and pressure fields, from which any number of indicators may be extracted. To date, most computational studies have assumed idealized vascular geometries. While the prediction of the flow through such geometries may yield useful insights into important physical processes, the results cannot be extrapolated with much confidence to the actual case which is characterized by a particularly irregular topology that gives rise to complex flow features. One of the most important recent advancements has therefore been the development of techniques to derive the geometry and flow boundary conditions from medical imaging data.

At present, an active area of research in blood flow is the study of unsteady flows in blood vessels with either a blockage or dilation. The partial obstruction of arteries due to a stenosis is one of the most frequent anomalies in blood circulation. It is well known that, once such an obstruction is formed, the blood flow is significantly altered and fluid dynamic factors play an important role as the stenosis continues to develop [6]. On the other hand, an aneurysm is a balloon-like dilation found on the walls of a blood vessel. An untreated aneurysm may burst under sustained internal pressure, causing death or grave disability. Hemodynamic factors such as velocity, pressure or shear stress may contribute to the growth of an aneurysm in a blood vessel. Thus far, the specific role of these factors is not yet well understood. The ability to describe the flow through a stenosed or dilated vessel would provide the possibility of diagnosing these diseases in their earlier stages.

Due to the complexity of modeling blood flow in this area, most previous studies have considered idealized vascular geometries, and assumed blood as a Newtonian fluid. Recently, the trend has shifted towards using realistic three-dimensional vascular geometries obtained from various medical imaging techniques. Younis et al. [7] considered blood flow in an exact replica of a stenosed human carotid artery. Their aim was to determine whether their computational model could capture the complex flow features shown in an experimental study. Neofytou and Tsangaris [8] used a CFD model to investigate the effects of different blood rheological models in analyzing flows through a stenosis and an abdominal aortic aneurysm (AAA). They showed significant differences between simulating blood as Newtonian and non-Newtonian fluids. Axisymmetric and asymmetric 3D stenotic models were used by Long et al. [9] to study the flow pattern and the wall shear stress
(WSS) distribution in the poststenotic region. Their results showed a complexity of the flow patterns in this region. The phenomenon of wall deformability was taken into account in the study of Tang et al. [10] where a 3D computational model with fluid-wall interaction was introduced to quantify related critical flow attributes.

Recently, Valencia and Solis [11] examined blood flow dynamics in a saccular aneurysm model with elastic walls of the basilar artery. They found the shear stress on the aneurysm wall and its deformation is dependent on the wall thickness and the elastic or hyperelastic wall model. Oshima et al. [12] employed the finite-element method to study the flow in cerebral aneurysm. Their geometrical model was derived from computed tomography data. The finite-element method was also used by Kumar and Naidu [13] to perform 2D axisymmetric simulations in aneurysms models with $0-75 \%$ dilation. Their results examined the sensitivity of various flow parameters to dilation height.

The distribution of WSS in coronary arteries is thought to be a significant factor in the onset of coronary heart disease [14]. In recent years, there has been controversy as to whether high, low or oscillating WSS is implicated in the development of atheroma [15]. Despite the clinical importance of atherosclerosis in the coronary arteries, there have been relatively few studies in this area due to the complex geometries and small size. Most studies have been on the left main coronary bifurcation [16], although the right coronary artery (RCA) has been attracting some recent interest. Myers et al. [14] used CFD techniques to study flow and WSS patterns in an anatomically faithful model of a human RCA. Recently, Johnston et al. [17] examined pulsatile blood flow through four different RCAs using several non-Newtonian blood models.

The aim of this study is three-fold: firstly, to investigate the variation in WSS in an artery with a stenosis or aneurysm at different flow rates and degrees of severity; secondly, to compare the various blood models and hence quantify the differences between the models and judge their significance; and lastly, to determine whether the use of the Newtonian blood model is appropriate over a wide range of shear rates.

\section{Analysis and modeling}

\subsection{Governing equations}

The blood flow is assumed to be laminar and incompressible and therefore the Navier-Stokes equations for 3D incompressible flow are given by: 


$$
\begin{gathered}
\nabla \cdot V=0 \\
\rho\left(\frac{\partial V}{\partial t}+V \cdot \nabla V\right)=-\nabla \cdot \tau-\nabla p
\end{gathered}
$$

where $V$ is the three-dimensional velocity vector, $p$ pressure, $\rho$ density, and $\tau$ the shear stress term.

We considered four different non-Newtonian blood flow models and compared the results obtained with that from the simple Newtonian model in this study. The effects of these models on the flow field and the WSS in the vicinity of the stenosis and aneurysm are examined. These models are given in the following subsection.

\subsubsection{Blood models}

\section{Newtonian model}

$$
\mu=0.00345 \mathrm{~Pa} \cdot \mathrm{s} .
$$

2. Power Law model

$$
\mu=\mu_{0}(\dot{\gamma})^{n-1}
$$

where $\mu_{0}=0.01467$ and $n=0.7755$.

3. Casson model

$$
\mu=\frac{\left[\sqrt{\tau_{y}}+\sqrt{\eta|\dot{\gamma}|}\right]^{2}}{|\dot{\gamma}|}
$$

where $\eta=\eta_{0}(1-H)^{-2.5}$ and $\tau_{y}=0.1(0.625 H)^{3}$ with $\eta_{0}=0.0012$ $\mathrm{Pa} \cdot \mathrm{s}$ and $H=0.37$.

4. Carreau model

$$
\mu=\mu_{\infty}+\left(\mu_{0}-\mu_{\infty}\right)\left[1+(\lambda \dot{\gamma})^{2}\right]^{(n-1) / 2}
$$

where $\mu_{0}=0.056 \mathrm{~Pa} \cdot \mathrm{s}, \mu_{\infty}=0.00345 \mathrm{~Pa} \cdot \mathrm{s}, \lambda=3.313 \mathrm{~s}$ and $n=0.3568$.

5. Generalized Power Law model

$$
\mu=\lambda|\dot{\gamma}|^{n-1},
$$

where

and

$$
\begin{gathered}
\lambda(\dot{\gamma})=\mu_{\infty}+\Delta \mu \exp \left[-\left(1+\frac{|\dot{\gamma}|}{a}\right) \exp \left(\frac{-b}{|\dot{\gamma}|}\right)\right], \\
n(\dot{\gamma})=n_{\infty}-\Delta n \exp \left[-\left(1+\frac{|\dot{\gamma}|}{c}\right) \exp \left(\frac{-d}{|\dot{\gamma}|}\right)\right],
\end{gathered}
$$

where $\mu_{\infty}=0.00345, n_{\infty}=1.0, \Delta \mu=0.25, \Delta n=0.45, a=50, b=3$, $c=50$, and $d=4$.

These models have been created by various researchers (Walburn and Schneck [18], Cho and Kensey [19], Fung [20], Ballyk et al. [21]), by fitting the input parameters in these models to experimental data for blood viscosity measured at certain shear rates.

\subsection{Geometry}

The flow geometry comprises a tube of diameter $D$ and can be divided into three regions, the inlet, the deformed, and the outlet region. In the case of the stenosis, the lengths of these regions are $4 D, 2 D$ and $20 D$, respectively. For the aneurysm, these are $4 D, 4 D$ and $18 D$, respectively. The radius of the undeformed inlet and outlet is $R_{0}=D / 2$.

In the case of the stenosis, the radius of the constricted region is given by:

$$
R=R_{0}\left[1-\left(\frac{R_{0}-R_{\min }}{R_{0}}\right)\left(\frac{1-\cos (\pi x / D)}{2}\right)^{2}\right], \quad 0 \leq x \leq 2 D,
$$

where $R_{\min }$ is the minimum radius at the centre of the stenosis. In this study, three different degrees of stenosis, $20 \%, 50 \%$ and $80 \%$, were used.

In the case of the aneurysm, the radius of the dilated region is given by:

$$
R=R_{0}+\left(a-R_{c}+\sqrt{R_{c}^{2}-(b / 2-x)^{2}}\right), \quad 0 \leq x \leq b,
$$

where $x$ is measured from the start of the dilated region, $a$ is the maximum width of the dilated segment, and $R_{c}=\left[a^{2}+(b / 2)^{2}\right] / 2 a$.

For this study, three different values, 0.25, 0.4 and 0.55 , of $a$ were used.

\subsection{Assumptions and boundary conditions}

It is assumed that the arterial walls are rigid and no-slip condition is imposed at the walls. At the outlet, stressfree conditions are applied and the pressure is set to zero. Finally, the velocity profile at the inlet is regarded to be that of fully developed flow in a straight tube and can be derived analytically for both the Newtonian and the Power Law fluids [8]. The forms for the Newtonian flow and the non-Newtonian flows are: 


$$
u=\bar{u}\left[1-\left(\frac{r}{R_{0}}\right)^{2}\right], \quad 0 \leq r \leq R_{0}
$$$$
\text { and } u=\bar{u}\left(\frac{3 n+1}{n+1}\right)\left[1-\left(\frac{r}{R_{0}}\right)^{\frac{n+1}{n}}\right], \quad 0 \leq r \leq R_{0} \text {, }
$$

respectively, where $u$ is the velocity component in the $x$-•direction. In transient flow, the pulsatile flow at the inlet is given by a time varying forcing function given in [22]. This forcing function was scaled to yield a maximum inflow velocity of $\bar{u}$ with a heart rate of approximately 60 beats per minute.

\subsection{Solution methodology}

The governing equations are highly nonlinear and must be solved numerically using techniques of CFD. In this study, these equations are solved using the finite element method as implemented by COMSOL (COMSOL Inc., Los Angeles, CA, USA). The flow geometries for the stenosis and aneurysm were first created using Matlab (MathWork Inc., Natick, MA, USA). Then a finite element mesh was placed on these geometries. Briefly, an inlet plane of the artery is meshed in 2D using triangles and this mesh is extruded along the centerline of the artery to create a 3D mesh consisting of hexadrel elements. The mesh used for all computations consisted of 9708 elements and 15,048 nodes for the stenosis and 17,696 elements and 27,132 nodes for the aneurysm as shown in Figure 1.
The governing equations were solved completely using the boundary conditions for fully developed flow, Equations (10) and (11), at the inlet along with the pulsatile forcing function for the transient case.

\section{Results and discussion}

Transient simulations were performed using all five models given above. Three different degrees of stenosis were used namely 20\%,50\% and 80\% and maximum dilated widths of $25 \%, 40 \%$ and $55 \%$ were examined for the aneurysm.

The distribution of the WSS is one of the most important hemodynamic parameters due to its direct relevance in artherosclerosis formation. Figure 2 shows the distributions of maximum shear stress for various degrees of severity of the stenosis for all models. It is evident that WSS increases with increasing severity. All models show close agreement with the Newtonian model except for the Power Law model. At 50\% stenosis, the WSS predicted by this model is significantly lower than the rest. Figure 3 shows the distribution of WSS along the geometry at various times. Maximum shear stresses are reached just before the throat of the stenosis. The magnitude of this value increases with higher flow rates. This peak is followed by a negative value indicating the presence of backflow. Further downstream, the WSS steadily regains its undisturbed value.

The maximum WSS for various degrees of dilation of an aneurysm is displayed in Figure 4 for all models. There is less agreement between the models in this case. Only the

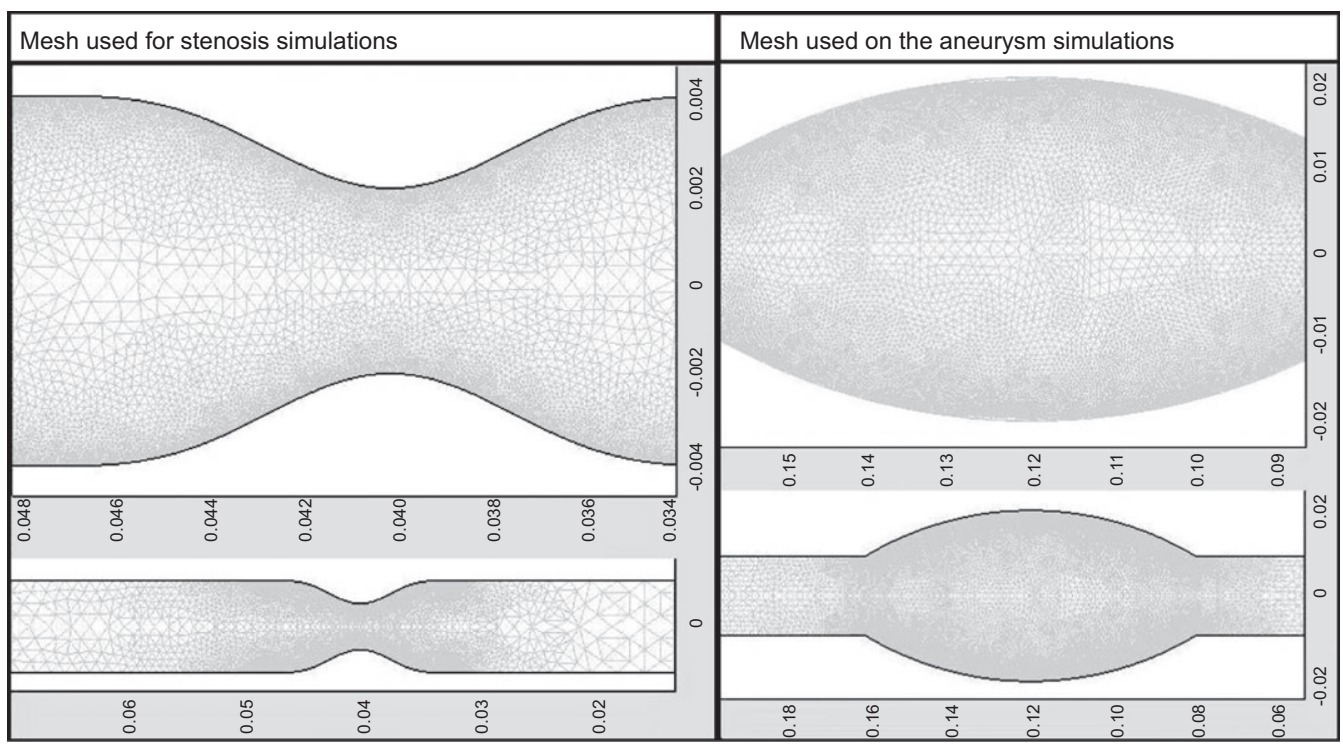

Figure $13 \mathrm{D}$ grid for stenosis and aneurysm. 


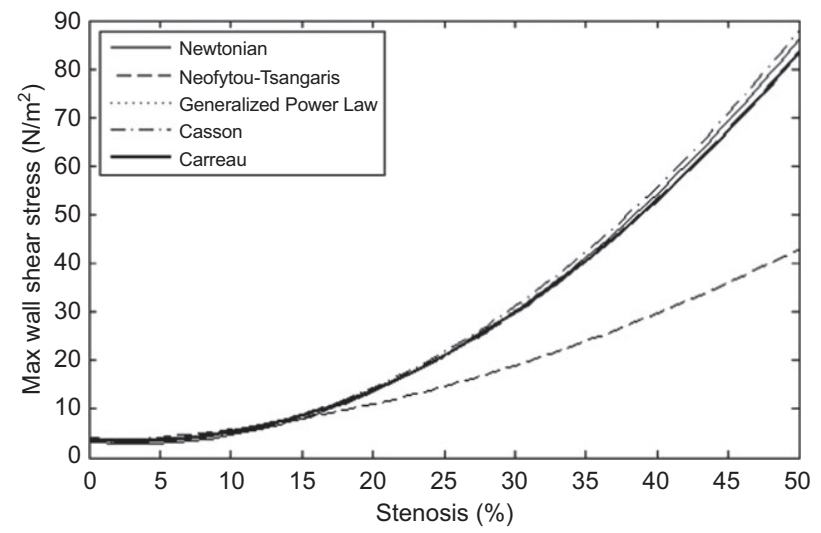

Figure 2 WSS vs. percent stenosis for various models, with 0.11196 $\mathrm{m} / \mathrm{s}$ inflow rate.

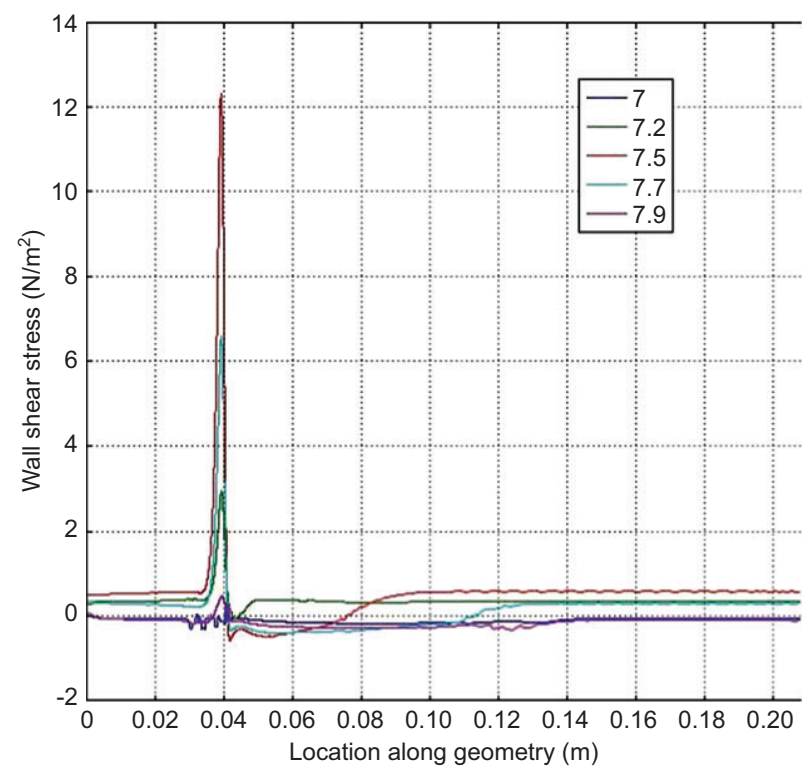

Figure 3 WSS for $50 \%$ stenosis pulsatile Generalized Power Law model at various time intervals, with $0.11196 \mathrm{~m} / \mathrm{s}$ max inflow rate.

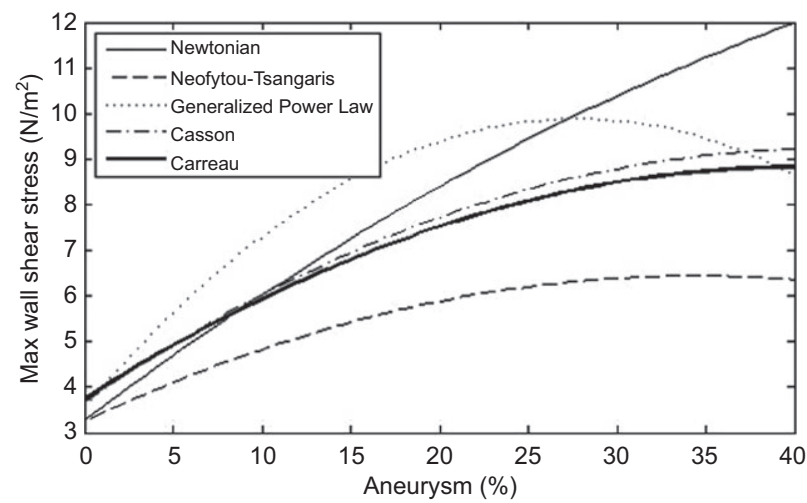

Figure 4 WSS vs. percent dilation for various models, with 0.11196 $\mathrm{m} / \mathrm{s}$ inflow rate.
Casson and the Carreau models are comparable throughout. The Power Law model gives a much lower $\tau_{W}^{\max }$ value because it exhibits a lower viscosity at the throat of the stenosis where the shear stress is high. As the flow rate increases, these WSS differences from various models become more prominent indicating significant differences in model behavior.

Transient simulations were performed using the Generalized Power Law model for both the stenosis and aneurysm. Each simulation was from $t=0-10.0 \mathrm{~s}$, yielding a heart rate of approximately 60 beats per minute.

Figure 5 shows the distribution of maximum WSS with shear rate in a stenosis. Again, WSS increases with increasing shear rate with the Power Law model deviating significantly from the rest.

Figure 6 displays the maximum WSS for increasing shear rate in an aneurysm. There is less agreement between the models at higher shear rates with the Power Law model showing the least agreement.

In the case of pulsatile flow through the aneurysm, the simulation was performed for $10 \mathrm{~s}$ using the Generalized Power Law model. The distribution of the WSS for the

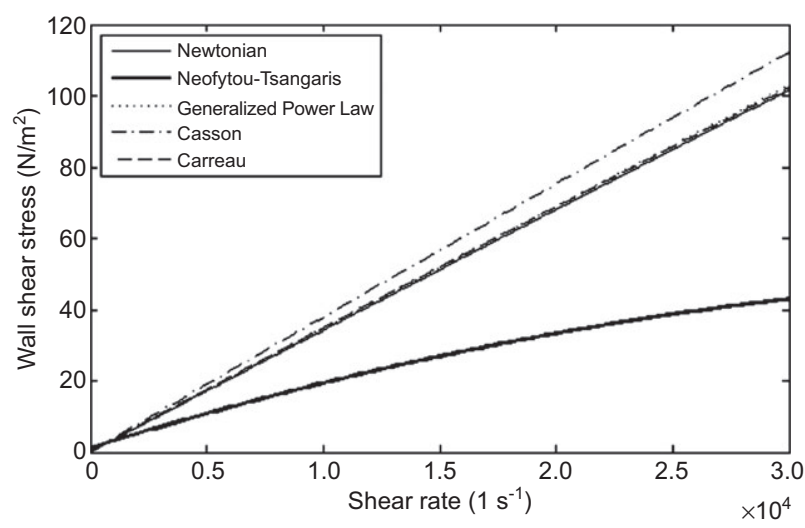

Figure 5 WSS vs. shear rate in a stenosis.

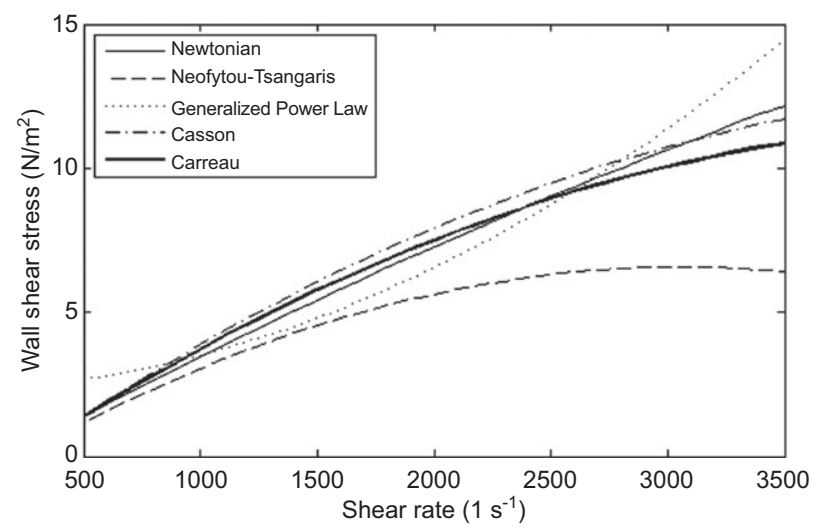

Figure 6 WSS vs. shear rate in an aneurysm. 


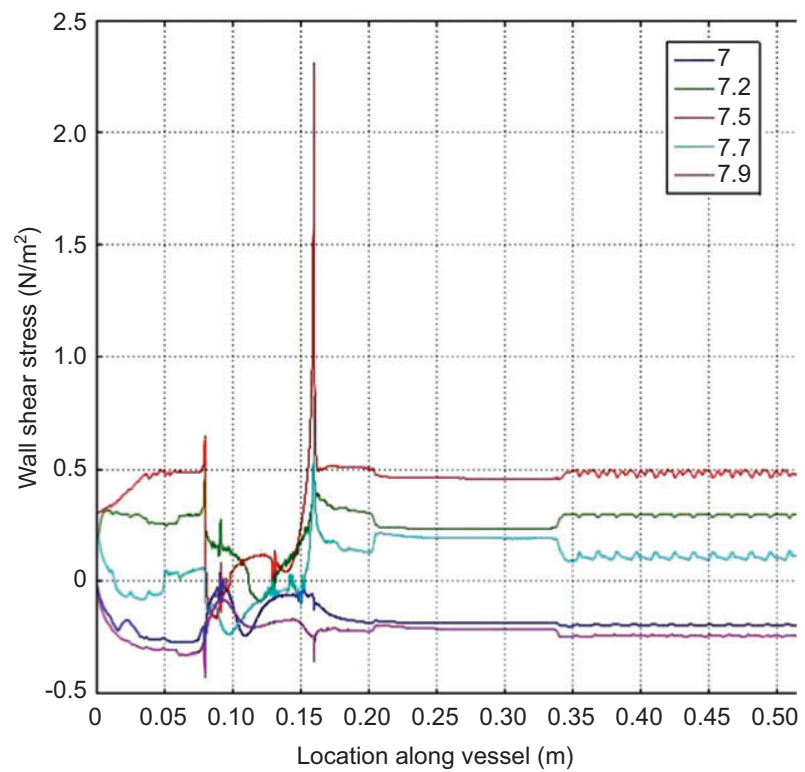

Figure 7 WSS for $55 \%$ aneurysm pulsatile Generalized Power Law model at various time intervals, with $0.13625 \mathrm{~m} / \mathrm{s}$ max inflow rate.

Generalized Power Law model is shown in Figure 7. The WSS drops as the flow enters the aneurysm and reaches a peak at the end of the dilated segment. This peak value increases with increasing flow rate. The negative WSS values indicate the presence of a recirculation region. As the flow exits the aneurysm, the WSS gradually regains its undisturbed value.

It was observed in the case of the stenosis that the maximum WSS values are produced at mid-cycle corresponding to the peak inflow velocity. However, the maximum pressure is attained just before mid-cycle and the minimum pressure towards the end of the cycle. This maximum pressure is excessively high. It is not clear why this is so and further investigation is planned. The streamline patterns at various points of the cardiac cycle for both the stenosis and aneurysm show the formation and reformation of the recirculation regions corresponding to the oscillatory nature of the pulsatile flow.

In all cases, for both the stenosis and the aneurysm, the flow field and the WSS changed significantly as the degree of abnormality increased. The recirculation regions become larger progressively and the WSS generally increases, especially for the stenosis.

\section{Conclusions}

A study of the effects of modeling blood flow through a stenosis and an aneurysm using five different blood rheological models was presented. The flow field and
WSS distributions produced by each model were investigated for various flow rates and degrees of abnormality. The results showed that there are significant differences between simulating blood as a Newtonian or non-Newtonian fluid. It was found that the Newtonian model is a good approximation in regions of mid-range to high shear but the Generalized Power Law model provided a better approximation of WSS at low shear.

These conclusions are presented under the assumption that the arterial walls are rigid and zero pressure is assumed at the outlet. A more realistic simulation would include elastic walls and incorporate the effects of upstream and downstream parts of the circulatory system into the boundary conditions. This is a long term objective of this study.

\subsection{Current limitations and future research}

Although there have been significant developments in blood flow modeling within the last decade, there remains some important limitations which can and should be overcome within the next decade to improve and extend the applications of CFD. Most notable are the following:

1. Most CFD models make the simplifying assumptions of rigid walls, Newtonian rheology, and fully developed flowboundary conditions. Thereis stillsome controversy regarding the relative impact of these assumptions on the prediction of hemodynamic indicators. Therefore, an important goal for the next decade is to try to assess the significance of these assumptions in the context of the clinical or biological problems being considered.

2. In most computing studies there is an explicit (or, more commonly, implicit) assumption of normal and periodic flow conditions. While some studies have investigated the effect of gross physiological changes on large artery flow dynamics, the effects of normal physiological variations in heart and flow rate remain unclear. Also, as it becomes possible to consider answering "what if” questions with CFD models, (e.g., what if a stent is placed here?), some careful consideration must be given to how the inflow and outflow boundary conditions will respond to the predicted hemodynamic alterations.

3. Current numerical methods are based on continuum models on the macroscale and do not incorporate biological phenomena on the molecular and cellular scales so important in disease processes. Furthermore, most CFD simulations are performed for a few seconds duration, but diseases develop over years. Mathematical models and numerical methods that span the physical and temporal scales in biological systems are needed. 


\section{References}

[1] Friedman MH, Bargeron CB, Duncan DD. J. Biomech. Eng.-T ASME 1992, 114, 317-320.

[2] Taylor CA, Hughes T, Zarine C. Comput. Phys. 1996, 10, 224-232.

[3] Perktold K, Peter RO, Resch M, Langs G. J. Biomed. Eng. 1991, 13, 507-515.

[4] Steinman DA. Ann. Biomed. Eng. 2002, 30, 483-497.

[5] Bale-Glickman J, Selby K, Saloner D, Savas O. J. Biomech. Eng.-TASME 2003, 125, 218-222.

[6] Pontrelli G. Proc. Inst. Mech. Eng. H. 2001, 215, 1-10.

[7] Younis BA, Spring S, Olaf Neumann S, Weigand B. Appl. Math. Model. 2007, 31, 2599-2609.

[8] Neofytou P, Tsangaris S. Int. J. Numer. Meth. Fl. 2006, 51, 489-510.

[9] Long Q, Xu XY, Ramarine KV, Hoskins P. J. Biomech. 2001. 34, $1229-1242$.

[10] Tang D, Yang C, Kobayashi S, Ku DN. Appl. Numer. Math. 2001, 38, 49-68.

[11] Valencia A, Solis F. Comput. Struct. 2007, 84, 1326-1337.
[12] Oshima M, Torii R, Kobayashi T, Taniguchi N, Takagi K. Comput. Method. Appl. M. 2001, 191, 661-671.

[13] Kumar BVR, Naidu KB. Comput. Biomed. Res. 1996, 2, 119-139.

[14] Myers JG, Moore JA, Ojha M, Johnston KW, Ethier CR. Ann. Biomed. Eng. 2001, 29, 109-120.

[15] Giddens DP, Zarins CK, Glagov S. Appl. Mech. Rev. 1990, 43, S98-S102.

[16] Perktold K, Hofer M, Rappitsch G, Loew M, Kuban BD, Friedman MH. J. Biomech. 1998, 31, 217-228.

[17] Johnston BM, Johnston PR, Corney S, Kilpatrick D. J. Biomech. 2006, 39, 1116-1128.

[18] Walburn FJ, Schneck DJ. Biorheology 1976, 13, 201-210.

[19] Cho YI, Kensey KR. Biorheology 1991, 28, 241-262.

[20] Fung YC. Biomechanics: Mechanical Properties of Living Tissues, 2nd ed. Berlin: Springer, 1993.

[21] Ballyk PD, Steinman DA, Ethier CR. Biorheology 1994, 31, 565-586.

[22] Holdsworth DW, Norley CJD, Frayne R, Steinman DA, Rutt BK. Physiol. Meas. 1999, 20, 219-240. 